\title{
HIGHLIGHTS
}

COMBINATION THERAPIES

\section{New prognostic factor for brain metastasis from ovarian cancer}

Ovarian cancer is one of the most

challenging solid tumors to treat. Although

optimal cytoreduction and adjuvant

therapy can improve outcomes, this

disease is still associated with high rates

of relapse and mortality. Historically,

the central nervous system (CNS)

is considered an uncommon site of

metastatic relapse; however, owing to the

improved survival as a result of treatment

advances, the likelihood of patients

presenting with brain metastasis from

ovarian cancer is increasing. Therefore,

identification of predictive and prognostic

markers are important goals of ovarian

cancer management.

Sehouli and coauthors undertook a multicenter retrospective analysis of 4,077 patients with histologically confirmed epithelial ovarian cancer, 74 of whom had brain metastases. The median time interval between the primary ovarian cancer diagnosis and brain metastases was 28.8 months. About $87 \%$ of women had FIGO (International Federation of Gynecology and Obstetrics) stage III or IV disease at primary diagnosis, and 58\% had grade 3 tumors. Most women (78.4\%) presented with multiple metastatic lesions at the time of CNS relapse.
Of the 74 patients with brain metastases, 21 received trimodality therapy consisting of surgery, radiotherapy and chemotherapy, 14 were treated with surgical resection and radiotherapy, and the remaining patients had cytoreduction or chemotherapy. In multivariate analysis, five factors had a significant survival impact after diagnosis of brain metastases. Those with multiple lesions had a fourfold risk of dying compared with patients who had a single lesion. A high Karnofsky performance status $(60-80 \%)$ at the time of brain relapse was significantly associated with longer survival.

Tumor grading and FIGO stage also affected outcomes. Paradoxically, patients with low grade ( 1 or 2 ) tumors had a significantly shorter survival compared with those with grade 3 disease, and patients with an advanced stage at initial diagnosis had a better survival after diagnosis of brain metastases than those with stage I or II. The investigators commented that potential selection bias in this poor prognostic group cannot be excluded.

No benefit in survival after diagnosis was shown in patients receiving multimodal therapies compared with a single modality. An interesting finding of this study is that patients with platinum sensitivity had a significantly better survival than those with platinum-resistant disease.

"An interesting and novel finding of the current study is the fact that platinum sensitivity had a highly significant positive impact on survival from

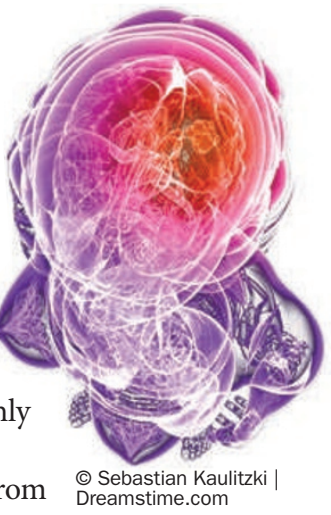
the diagnosis of brain metastasis," conclude the researchers. The choice of systemic therapy is also based largely on the differentiation of patients who respond to platinum-based therapy and those who do not. As this observation is the single most important prognostic factor, this finding should be considered when using a multimodal approach for managing patients who have brain metastases from ovarian cancer.

\section{Lisa Hutchinson}

Original article Sehouli, J. et al. Prognostic role of platinum sensitivity in patients with brain metastases from ovarian cancer: results of a German muticenter study. Ann. Oncol. 21, 2201-2205 (2010) 